\title{
New multiphase choke correlations for a high flow rate Iranian oil field
}

\author{
M. Safar Beiranvand ${ }^{1}$, P. Mohammadmoradi ${ }^{2}$, B. Aminshahidy ${ }^{1}$, B. Fazelabdolabadi ${ }^{1}$, and \\ S. Aghahoseini ${ }^{3}$ \\ ${ }^{1}$ Institute of Petroleum Engineering, College of Engineering, University of Tehran, Tehran, Iran \\ ${ }^{2}$ Department of Chemical and Petroleum Engineering, Sharif University of Technology, Tehran, Iran \\ ${ }^{3}$ Department of Petroleum Engineering, Azad University of Kharg Island, Kharg Island, Iran
}

Correspondence to: M. Safar Beiranvand (mahmoud.beiran@ut.ac.ir)

Received: 18 August 2011 - Revised: 8 May 2012 - Accepted: 20 May 2012 - Published: 22 June 2012

\begin{abstract}
The multiphase flow through wellhead restrictions of an offshore oil field in Iran is investigated and two sets of new correlations are presented for high flow rate and water cut conditions. The both correlations are developed by using 748 actual data points, corresponding to critical flow conditions of gas-liquid mixtures through wellhead chokes. The first set of correlations is a modified Gilbert equation and predicts liquid flow rates as a function of flowing wellhead pressure, gas-liquid ratio and surface wellhead choke size. To minimize error in such condition, in the second correlation, free water, sediment and emulsion (BS \& W) is also considered as an effective parameter. The predicted oil flow rates by the new sets of correlations are in the excellent agreement with the measured ones. These results are found to be statistically superior to those predicted by other relevant published correlations. The both proposed correlations exhibit more accuracy (only $2.95 \%$ and $2.0 \%$ average error, respectively) than the existent correlations. These results should encourage the production engineer which works at such condition to utilize the proposed correlations for future practical answers when a lack of available information, time, and calculation capabilities arises.
\end{abstract}

1

\section{Introduction}

Generally almost all flowing wells utilize wellhead chokes in order to regulate the flowing rate, prevent water and gas coning and sand problems, as well as valves installed for safety purposes. As a major factor of killing the producer well, the inaccurate flow rate predictions may inevitably lead towards gas or water coning, sand entry and excessive pressures at the separator. Larger amount of produced water contributes to high operating costs and is a major environmental concern for oil production (Jin and Wojtanowicz, 2010).

The most of published multiphase flow correlations are highly empirical (Mesallati et al., 2000). Several correlations have been offered for describing critical and subcritical multi-phase flow through wellhead chokes (Al-Attar, 2009). Majority of such correlations are based on limited ranges of flow variables. According to quality and scope of data upon which they are based, the validity of those correlations is limited. The most popular correlation was developed by Gilbert (1954) but it is valid for critical flow occurring when the upstream pressure of the choke is at least $70 \%$ higher than the downstream pressure or when the ratio of downstream pressure to upstream pressure is equal to 0.588 (Ghareeb and Shedid, 2007). The most popular correlation was developed by Gilbert (1954) but it is valid for critical flow occurring when the upstream pressure of the choke is at least $70 \%$ higher than the downstream pressure (Ghareeb et al., 2007). However, other researchers proposed various correlations rather than Gilbert's equation in the literature.

Flow through the wellhead chokes mainly fall into two critical and subcritical conditions.

Within this context, the critical flow condition refers to the state which the flow rate reaches a maximum value that is independent of a pressure drop applied across the choke and changes in pressure downstream of the choke do not affect the flow rate. Hence, wellhead chokes are commonly 
Table 1. Equation coefficient for different correlations and their accuracy (General form: $\left.Q=A \frac{P_{\mathrm{wh}} S^{B}\left(1-\frac{\mathrm{BS} \& \mathrm{~W}}{100}\right)^{D}}{\mathrm{GLR}^{C}}\right)$.

\begin{tabular}{lllllll}
\hline \multirow{2}{*}{ Correlation } & \multicolumn{5}{c}{ Empirical Coefficient } & \\
\cline { 2 - 5 } & $A$ & $B$ & $C$ & $D$ & Error \% & Absolute Error \% \\
\hline This Work $^{1}$ & 0.0328 & 2.275 & 0.586 & 0 & 2.95 & 12.1 \\
This Work $^{2}$ & 0.0382 & 2.151 & 0.5154 & 0.52965 & 2 & 9.8 \\
Gilbert & 0.1 & 1.89 & 0.546 & 0 & 11.26 & 18.37 \\
Ros & 0.574 & 2 & 0.5 & 0 & 13.45 & 19.2 \\
Baxendell & 0.1046 & 1.93 & 0.546 & 0 & 32.1 & 33 \\
Achong & 0.2618 & 1.88 & 0.65 & 0 & & \\
\hline
\end{tabular}

Table 2. Range of data used for the correlations.

\begin{tabular}{llllll}
\hline & $\begin{array}{l}\text { Flow Rate } \\
(\mathrm{bbl} / \text { day) (STB/Day) }\end{array}$ & $\begin{array}{l}\text { GLR } \\
(\mathrm{SCF} / \mathrm{STB})\end{array}$ & $\begin{array}{l}\text { Choke Size } \\
(1 / 64) \text { in }\end{array}$ & $\begin{array}{l}\text { Wellhead Pressure } \\
(\text { Psi) }\end{array}$ & $\begin{array}{l}\text { BS\&W } \\
\text { (Percent) }\end{array}$ \\
\hline Range & $3000-24000$ & $80-260$ & $16-40$ & $1400-12000$ & $0.1-30$ \\
\hline
\end{tabular}

operated under critical flow conditions in order to isolate the reservoir from pressure variation introduced by surface equipment. Empirical correlations are mainly used for critical flow condition. In this paper, production data from offshore field, in Iran, were used to generate two sets of new correlations for multiphase flow through wellhead surface choke.

\section{Backgrounds}

In 1954, Gilbert proposed his correlation relating surface production with the wellhead choke size as follows:

$Q=A \frac{P_{\mathrm{wh}} S^{B}}{\mathrm{GLR}^{\mathrm{C}}}, A=0.1, \quad B=1.89, \quad C=0.546$

Where $Q$ is gross liquid rate (bbl/day), GLR is gas-liquid ratio (MSCF/STB), $P_{\mathrm{wh}}$ is well (or tubing) head pressure (psig) and $S$ is bean size $(1 / 64)$ inch.

In 1960, Ros developed a very similar correlation to Gilbert (1954) correlation but with different correlating exponents as follows:

$Q=A \frac{P_{\mathrm{wh}} S^{B}}{\mathrm{GLR}^{C}}, A=0.574, \quad B=2.0, \quad C=0.5$

Another Gilbert form correlation was presented by Achong in 1961:

$Q=A \frac{P_{\mathrm{wh}} S^{B}}{\mathrm{GLR}^{C}}, A=0.2618, B=1.88, C=0.65$,

There were many other correlations to predict flow rates in the critical region. Some of them were similar to the Gilbert correlation, but with different constants and exponents. One of them was proposed by Baxendell (1958):

$Q=A \frac{P_{\mathrm{wh}} S^{B}}{\mathrm{GLR}^{C}}, \quad A=0.104, \quad B=1.93, \quad C=0.546$
These correlations are tabulated in Table 1.

\section{Developments of new correlation and discussion}

\subsection{Levenberg-Marquardt algorithm}

Levenberg-Marquardt algorithm is a method for finding the minimum of a multivariable nonlinear function which has been applied as a standard method for solving the squares minimum problem for the nonlinear functions. LevenbergMarquardt algorithm (LMA) interpolates between GaussNewton algorithm (GNA) and gradient descent method. LMA algorithm is more resistant than GNA, means most times even if has been started very far from the extreme minimum, will gain the answer and of course for well-behaved functions and reasonable initial parameters, LMA is slightly slower than NGA.

Least squares problem includes finding $\boldsymbol{P}$ parameters vector for which the following cost function is minimized:

$S(\boldsymbol{P})=f^{T} f=\sum_{i=1}^{m}\left[f_{i}(\boldsymbol{P})\right]^{2}$

Like as the other numerical minimization algorithms, Levenberg-Marquardt algorithm is a repeated process. For beginning the minimization, the user must present an initial guess for $\boldsymbol{P}$ parameters vector. Most cases a standard unconscious guess such as $\boldsymbol{P}^{T}=(1,1,000,1)$ applies very well. In other cases, the algorithm applies just when the initial guess is close to the final answer somehow.

In each repeat step, $\boldsymbol{P}$ parameters vector is replaced with a new $p+q$ estimation. For achieving $q, f_{i}(p+q)$ functions are estimated as $f_{i}(p+q) \approx f(p)+J_{q}$ through their linearization, that $J$ is Jacobin of $f$ in $p$.

In a minimum of sum of $S$ squares, we have $\nabla_{q} S=0$, that upon the above linearization the following equation 


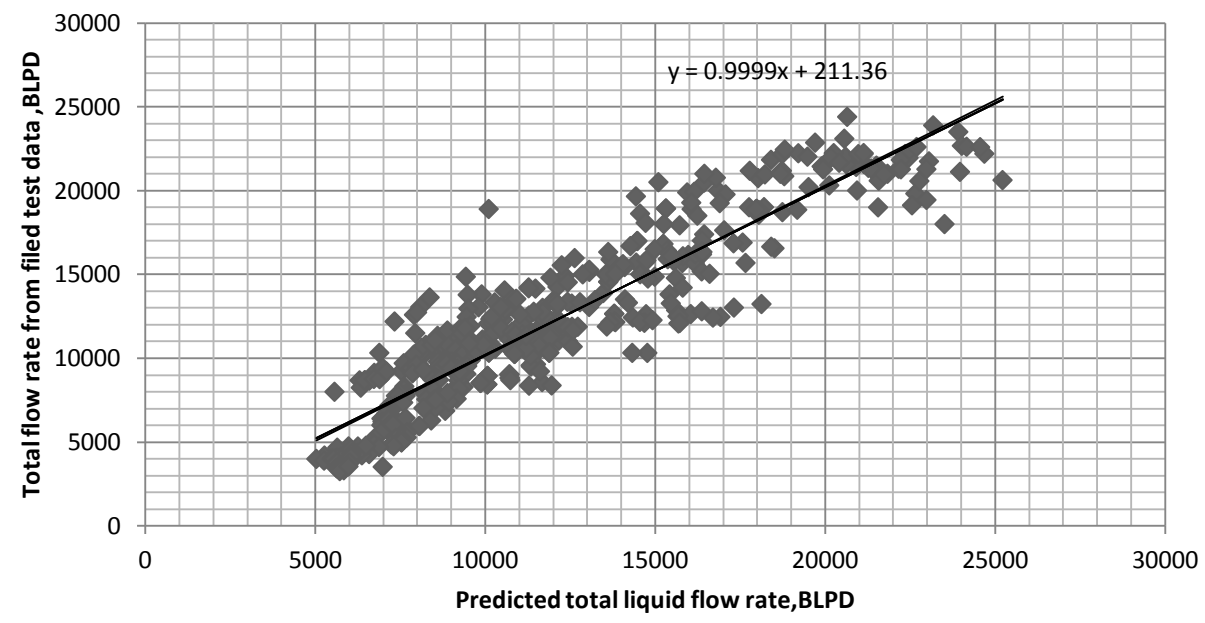

Figure 1. Predicted vs. measured flow rates for the first derived correlation.

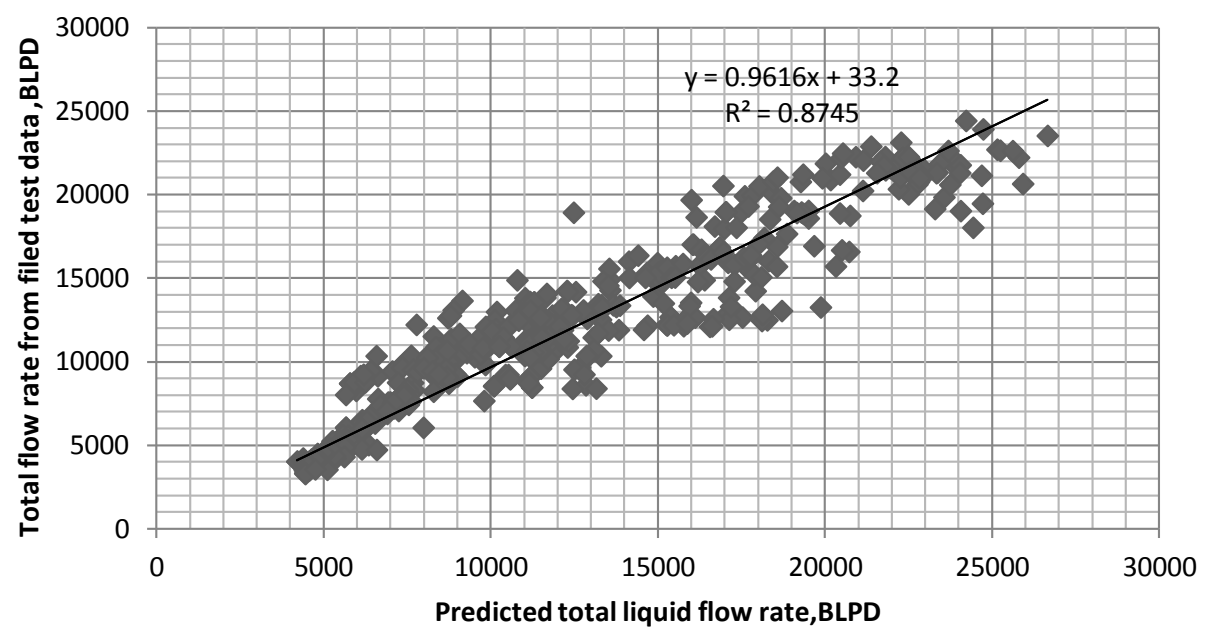

Figure 2. Predicted vs. measured flow rates for the second derived correlation.

is gained:

$\left(J^{T} J\right) q=-J^{T} f$

That $q$ may be gained thereof through reversing $J^{T} J$. LMA key is replacement of this equation with its "attenuated version"

$\left(J^{T} J+\lambda\right) q=-J^{T} f$

Nonnegative attenuation coefficient $\lambda$ is adjusted in each repeat. If the descent of $S$ was fast, we give a smaller value to which that closes the algorithm to GNA, but if a repeat exhibited inadequate descent, may ascend the $\lambda$ and take a step closer to gradient descent. A similar attenuation coefficient is observed in Tiknonov regularization which is useful for solving malfunctioned linear problems. If a recovered step length or descent of sum of squares for the last set of $\boldsymbol{P}$ parameters is lower than predetermined values, the repeat is finished and the last $\boldsymbol{P}$ parameter vector is deemed as the answer.

\subsection{Results and discussion}

748 production test data were collected for 10 wells, including the liquid flow rate, gas-liquid ratio, choke Size and wellhead pressure lied within the reign 3000-24000 (STB/day), 80-260 (SCF/STB), 16-40 (1/64th-inch) and 1400-12000 (Psi), respectively. Range of the data used is also tabulated in Table 2. The Levenberg-Marquardt Algorithm (LMA) is used for proposing the two sets of new correlations.

The first proposed correlation, a modified Gilbert equation based on regression analysis for the field is:

$Q=A \frac{P_{\mathrm{wh}} S^{B}}{\mathrm{GLR}^{C}}, A=0.0328, \quad B=2.275, \quad C=0.586$

The second newly-developed correlation considered a parameter which was not included in the previous correlations; free water, sediment and emulsion (BS \& W). This is in addition to other parameters appeared in Gilbert and other correlations, in order to minimize error in field condition, this 
correlation is:

$Q=A \frac{P_{\mathrm{wh}} S^{B}\left(1-\frac{\mathrm{BS} \& \mathrm{~W}}{100}\right)^{D}}{\mathrm{GLR}^{C}}, A=0.0382$,

$B=2.1510, C=0.5154, \quad D=0.5297$

where $P_{\text {wh }}$ is well head pressure (Psig), GLR is gas-liquid ratio ( $\mathrm{SCF} / \mathrm{STB}$ ), $Q$ is gross liquid flow rate (bbl/day), $S$ is choke size (64th of an inch) and finally, BS \& W, it includes free water, sediment and emulsion and is measured as a volume percentage of the production stream. As can be seen, in the Gilbert's formula, the unit of GLR is MSCF/STB whereas in the proposed correlation the unit is SCF/STB.

Generally adopted assumptions on flow conditions are:

- Critical multiphase flow occurs at a constant critical pressure ratio,

- Both phases flow with the same velocity in the throat,

- Friction is negligible, the flow behavior is dominated by changes in kinetic energy,

- Expansion of the gas phase in the throat is polytropic.

Regarding the above assumptions, the average and absolute errors are used for comparing correlations. These errors are statistical measurements of how far estimates or forecasts are from actual values and could be applied to any two pairs of numbers, where one set is "actual" and the other is an estimate, forecast or prediction.

Two types of errors are calculated using the equations as follow:

$e=\frac{q_{\text {test }}-q_{\text {correl }}}{q_{\text {test }}}$

$e_{\mathrm{abs}}=\left|\frac{q_{\mathrm{test}}-q_{\text {correl }}}{q_{\text {test }}}\right|$

The first proposed correlation, a modified Gilbert equation, has exhibited an average and absolute errors of $2.95 \%$ and $12.1 \%$ respectively, whereas the second proposed correlation has showed an average error of roughly $2.0 \%$, also absolute error of $9.8 \%$. The average and absolute errors of other types correlations are tabulated in Table 1. Figures 1 and 2 show a good agreement between the measured and the predicted data by the new correlations. As evident from Fig. 2, a very good accuracy of predicted production rates with correlation factor $\left(R^{2}\right)$ of 0.973 is obtained.

\section{Conclusions}

Based on 748 actual data points, the available choke correlations were reviewed and two sets of newly-established correlations were presented for an offshore field in Iran. The following conclusions are drawn:
1. The first proposed correlation, a modified Gilbert equation, has exhibited an average error of $2.95 \%$, whereas current correlations such as Gilbert and Ros represent average errors of $11.26 \%$ and $13.45 \%$, respectively.

2. The second newly-developed correlation considered a parameter which was not included in the previous correlations; free water, sediment and emulsion (BS \& W). This is in addition to other parameters appeared in Gilbert and other published correlations considered in this work, in order to minimize error in field condition. The second set of correlations exhibited an average error of $2.0 \%$.

3. The results strongly encourage the production engineer to utilize the proposed correlations for future practical answers in case of lack of information availability, time, and calculation capabilities arises.

4. Undoubtedly, presented correlations can be used, in order to controlling the water production as well as reach the optimum production for under consideration high flow rate Iranian oil field and similar cases.

\section{Appendix A}

\section{Nomenclature}

\begin{tabular}{ll}
\hline$A$ & Proportionality constant \\
$B$ & Bean or choke size exponent \\
bbl & Barrel \\
BS \& W & Basic Sediment \& Water \\
$C$ & Gas-oil ratio exponent \\
$D$ & Basic Sediment \& Water term exponent \\
$E$ & Temperature term exponent \\
$e$ & Error \\
$e_{\text {abs }}$ & Absolute error \\
$F$ & Well head pressure exponent \\
GLR & Producing gas-liquid ratio at standard \\
& conditions, SCF/STB \\
$P_{\text {wh }}$ & Well head pressure, psig \\
$Q$ & Gross liquid flow rate, bbl/day \\
$Q_{\text {correl }}$ & Calculated gross liquid flow rate \\
& from correlation, bbl/day \\
$Q_{\text {test }}$ & Measured gross liquid flow rate, bbl/day \\
$S$ & Choke or bean size, $1 / 64$ inch \\
SCF & Standard cubic foot \\
STB & Stock tank barrel \\
$T$ & Temperature, R \\
$T_{\mathrm{SC}}$ & Standard condition temperature, R \\
\hline &
\end{tabular}


Edited by: A. Barari

Reviewed by: three anonymous referees

\section{References}

Achong, I.: Revised Bean Performance Formula for Lake Maracaibo Wells, internal co. report, Shell Oil Co., Houston, TX, 1961.

Al-Attar, H. H.: New Correlations for Critical and Subcritical TwoPhase Flow Through Surface Chocks in High-Rate Oil Wells, SPE 120788, 2009.

Baxendell, P. B.: Producing Wells on Casing Flow - An Analysis of Flowing Pressure Gradients, AIME, 213, 202-207, 1958.

Economides, M. J., Hill, A. D., and Ehlig-Economides, C.: Petroleum Production Systems, Prentice Hall PTR, New Jersey, p. $229,1993$.
Ghareeb, M. and Shedid, A.: A New Correlation for Calculating Wellhead Production Considering Influence of Temperature, GOR, and Water-Cut for Artificially Lifted Wells, IPTC 11101, 2007.

Gilbert, W. E.: Flowing and Gas-Lift Well Performance, Dril. And Prod. Prac. API, 143, 127-154, 1954.

Jin, L. and Wojtanowicz, A. K.: Coning Control and Recovery Improvement Using in-Situ Water Drainage/Injection in Bottom Water-Drive Reservoir, SPE 129663, 2010.

Mesallati, A., Biznati, M., and Mansouri, N.: Multiphase-Flow choke correlations for Offshore Bouri Oil field, International Gas Union 21st World Gas Conference, 49 pp., Nice, France, 6-9 June, 2000.

Ros, N. C. J.: An Analysis of Critical Simultaneous Gas/Liquid Flow Through a Restriction and Its Application to Flow metering, Applied Science Research, 9, 374-388, doi:10.1007/BF00382215, 1961. 